\section{Loratadin bei Allergien}

\section{Lebensqualität verbessert}

\author{
Patienten mit Neurodermitis oder Urtikaria sind in ihrer Lebens- \\ qualität häufig stärker eingeschränkt als Patienten mit schweren \\ internistischen Erkrankungen. Nicht-sedierende Antihistaminika \\ wie Loratadin reduzieren bei diesen Erkrankungen vor allem den \\ Juckreiz und erhöhen die Lebensqualität.
}

A llergische Erkrankungen, die Haut wie auch die Schleimhäute z.B. der Nase betreffend, beeinträchtigen die Lebensqualität der Patienten. In welchem Ausmaß dies geschehen kann, verdeutlichen die Untersuchungen von Augustin und Mitarbeitern von der Forschungsgruppe für Psychosomatische Dermatologie und Lebensqualitätsforschung der Universitäts-Hautklinik Freiburg, die E. Schöpf (Freiburg) vorstellte. Die Ergebnisse zeigen, dass sich insbesondere Neurodermitispatienten in ihrer Lebensqualität stärker beeinträchtigt fühlen als Patienten nach Herzinfarkt oder anderen schweren internistischen Erkrankungen.

Auch bei Patienten mit Urtikaria fanden Augustin et al. ausgeprägte Einbußen der Lebensqualität sowohl auf körperlicher Ebene wie auch hinsichtlich des psychischen Befindens, der sozialen Kontakte oder der allgemeinen Zufriedenheit. Die Belastungsintensität war zwar nicht ganz so hoch wie bei den Neurodermitikern, aber vergleichbar mit denen bei Nahrungsmittelallergikern und lag deutlich über der von Patienten mit Medikamenten- oder Insektengiftunverträglichkeiten, Schuppenflechte oder Hautkrebs.

\section{Juckreiz gelindert}

Ziel einer adäquaten Therapie der Neurodermitis wie auch der chronischen Urtikaria ist daher die Verbesserung der Lebensqualität. Untersuchungsergebnissen z.B. von Studien mit Urtikariapatienten zufolge führt eine Behandlung mit Antihistaminika zu einer Verbesserung der Lebensqualität - eine symptomatische Gabe dieser Medikamente als erste Wahl sei daher gerechtfertigt, so Schöpf.
Die Verbesserung der Lebensqualität von Patienten mit Neurodermitis und Urtikaria durch Antihistaminika wird vor allem durch ihre juckreizlindernde Wirkung erreicht. Dieser Effekt ist eine Domäne der Antihistaminika, wobei sedierende Eigenschaften, wie sie den Vertretern der älteren Generation zu eigen ist, keine zwingende Vorausset- zung für die Juckreizlinderung sein müssen.

Wie R. U. Peter (Ulm) ausführte, konnte dies vor allem mit Loratadin (Lisino $\left.{ }^{\circledR}\right)$ als Vertreter der Antihistaminika der neuen Generation nachgewiesen werden. Peter: „Der damit eingeleitete Paradigmenwechsel führte zu einer raschen Verbreitung der nicht-sedierenden Antihistaminika auch zur Behandlung verschiedener pruriginöser Hauterkrankungen."

Loratadin hat eine hohe therapeutische Wirksamkeit bei praktisch fehlenden sedierenden Eigenschaften und auch sonst sehr guter Verträglichkeit und nimmt eine führende Stellung unter den neuen H1-Blockern ein. $\quad b k$

\section{Schöpf E, Peter RU}

Pressesymposium, veranstaltet von Essex Pharma, Hamburg, 2000.

\title{
Loratadin wirkt auch lokal im Darm
}

\section{Im akuten Schub einer Colitis ulcerosa sind verschiedene Entzündungsparameter erhöht. Eine Pilotstudie zeigt nun: Unter zusätzlicher Loratadingabe können Kortikoide eingespart werden.}

A llergien sind nicht selten mit einer Colitis ulcerosa assoziiert und bei vielen Colitispatienten hat ihre Erkrankung eine allergische Komponente. Nach M. Raithel (Erlangen) besteht ein wichtiger Pathomechanismus dieser entzündlichen Erkrankung in der Aktivierung von CD4+TH2-Lymphozyten. Dadurch kommt es zu einer vermehrten Ausschüttung u.a. von Histamin aus den Mastzellen der Darmschleimhaut, wodurch sich ein Teil der Symptome des akuten Schubes einer C. ulcerosa erklären lässt.

H1-Blocker wie Loratadin (Lisino $\left.{ }^{\circledR}\right)$ können die Krankheitssymptome lindern, so die Ergebnisse einer von Raithel in Erlangen durchgeführten Pilotstudie: 18 Patienten mit einer mindestens mittelschweren Colitis und einem vernachlässigbaren Atopiescore erhielten über sechs Monate neben der Standardmedikation (Prednisolon/5Aminosalicylsäure) zusätzlich $10 \mathrm{mg} / \mathrm{d}$ Loratadin oder Plazebo.
Die Kortisondosis wurde dabei im Verlauf der Behandlung reduziert. Dies konnte bei den mit Loratadin behandelten Patienten aber in weitaus stärkerem Ausmaß erfolgen als bei den mit Plazebo behandelten Patienten. Dies spiegelte sich auch in einem Rückgang antientzündlicher Parameter wie ECP unter Loratadin wieder.

Der Effekt des Antihistaminikums tritt erst nach ein bis zwei Behandlungsmonaten ein und, so Raithel, könnte durch Steigerung der Dosis auf 20 bis $30 \mathrm{mg}$ Loratadin/d noch gesteigert werden. Raithel: „Die Blockade des H1-Rezeptors scheint somit zusätzliche antientzündliche Effekte zu vermitteln, die durch die bisherige Standardtherapie mit einem Steroid und 5-Aminosalicylsäure noch nicht ausreichend erfasst werden."

\section{Raithel M}

Pressesymposium, veranstaltet von der Essex Pharma, Hamburg, 2000. 